\title{
Esophageal motility testing: The spastic truth
}

\author{
William Paterson MD FRCPC ${ }^{1}$, Paul C Adams MD², Editor-in-Chief
}

$\mathrm{D}^{\mathrm{r}}$ William Paterson is a Professor of Medicine at Queen's University (Kingston, Ontario) and the current President of the Canadian Association of Gastroenterology. He began his career in esophageal motility research at Harvard University (USA) with Dr Raj Goyal and has an ongoing Canadian Institutes of Health Research-funded program that focuses on basic esophageal physiology and pathophysiology. He also directs the gastrointestinal function laboratory at Hotel Dieu Hospital (Kingston, Ontario).

PA: You are called in to see a patient in the cardiology ward who has just had an angiogram and an angioplasty, but continues to have angina-like chest pain. The patient has been receiving proton pump inhibitor (PPI) therapy. The cardiologist told him/her that he/she has minimal heart problems (although he/she just had an angioplasty) and most likely a spastic esophagus. What is your approach to this common type of referral? If manometry is not readily available at your centre, can you comment on the use of endoscopy and barium stud-

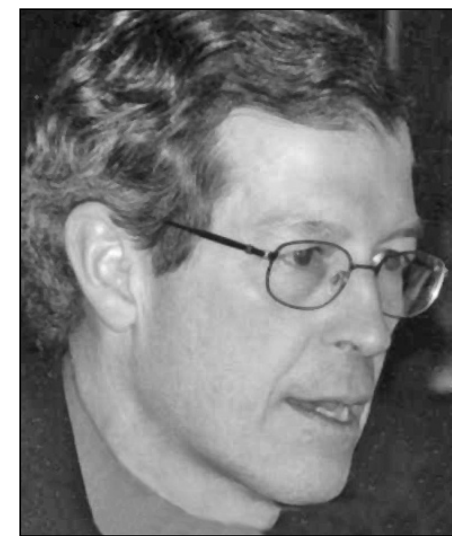

Dr William Paterson is a Professor of Medicine at Queen's University (Kingston, Ontario) and the current President of the Canadian Association of Gastroenterology antidepressant. Barium studies are of no value unless the patient has associated dysphagia. The yield on endoscopy is also very low. It is nice if you find reflux esophagitis in these patients because you then have a firm rationale for PPI therapy. However, in practice you will likely give a treatment trial for reflux irrespective of whether there is macroscopic esophagitis.

PA: Often these patients may have symptoms that have been more traditionally associated with angina such as radiation of pain down the arm, diaphoresis or pain during exercise. Could some of these patients have microvascular angina?

WP: Yes, microvascular angina or so-called cardiac syndrome $\mathrm{X}$ may be present in some of these patients. It is more common in woman. Usually, this syndrome is characterized by classic, exertionally related chest pain. Patients usually do not present with sporadic chest pain unrelated to exercise. I am, therefore, very reluctant to consider esophageal origin of the pain in patients that have typical exertional pain irrespective of the angiographic findings. In these patients, I usually ask the cardiologist WP: Actually, at least at our centre, referrals from cardiologists for patients with so-called noncardiac chest pain are no longer that common. We have noted a dramatic reduction in the number of patients who are referred to our centre with this problem, both in the inpatient and outpatient setting. I am not sure exactly why this is the case. It may be that family doctors and cardiologists realize that we are no longer very enthusiastic about pursuing various diagnostic tests in the workup of these patients. Since the Canadian Association of Gastroenterology and the American Gastroenterological Association guidelines $(1,2)$ on noncardiac chest pain and esophageal manometry were published approximately 10 years ago, we have seen a significant change in the referral pattern for esophageal motility studies. Whereas in the past, probably two-thirds of the patients undergoing manometry were referred because of noncardiac chest pain, that number has now decreased to less than $20 \%$. The majority are referred for evaluation of nonstructural dysphagia. This change in practice is due to our increasing realization that although esophageal manometry and provocative testing may provide us with some insight into the pathogenesis of the patient's chest pain, it lacks sensitivity and specificity and rarely guides us toward a specific therapy. More commonly, the approach being taken in these patients, once cardiac disease is excluded, is to treat empirically with either a PPI or a tricyclic to reconsider the possibility of microvascular angina.

PA: We have seen reports that patients can have manometric abnormalities with no symptoms and symptoms with no manometric abnormalities? How do you interpret these observations? WP: That is one of the basic problems with using manometry in patients with chest pain. There is rarely any direct correlation between the manometry abnormalities and the pain episodes. We do see a rare patient who has very prolonged duration contractions that seem to coincide with pain episodes. The usual high amplitude contractions seen in Nutcracker esophagus do not correlate with the pain. Many of us believe that this is because the hypertensive peristaltic contractions seen in Nutcracker esophagus may simply be a sign of hyperreactivity of the esophagus. Many of these patients have evidence of visceral sensory abnormality affecting the esophagus. The other reason for poor correlation with intraluminal manometry is that the 'muscle spasms' that we have always presumed were the cause of noncardiac chest pain, are actually occurring in the longitudinal smooth muscle. These contractions lead to shortening of the esophagus but do not produce a detectable change in the intraluminal pressure. A study by Balaban et al (3), using intraluminal ultrasound, has suggested that there are sustained contractions of the longitudinal muscle that correlate with certain pain episodes. We have also shown, in animal models, that acid 
in the lumen can produce a sustained reflex contraction of the longitudinal muscle.

PA: One of the most well accepted indications for manometry is before fundoplication. Is this still a strong recommendation? WP: Although virtually all antireflux surgeons will request esophageal manometry before proceeding with fundoplication, I am not sure if I would describe this as a well accepted indication. In the updated American Gastroenterological Association medical position statement on the clinical use of esophageal manometry published last year, it is stated that "manometry is possibly indicated for the pre-operative assessment of peristaltic function in patients being considered for anti-reflux surgery" (4). The main rationale for performing manometry before surgery is to rule out an unrecognized, severe, primary motor disorder. There are case reports of patients with a sclerodermatype esophagus, diffuse esophageal spasm or even achalasia who underwent antireflux surgery and then developed severe unremitting dysphagia subsequently. This can lead to quite disastrous results, sometimes leading to esophagectomy. However, there is now abundant evidence in the literature that most patients with significant peristaltic dysfunction do not have a significantly worse outcome after antireflux surgery, providing the patient does indeed have underlying reflux disease. My own view is that in patients with typical clinical and endoscopic evidence of reflux disease and no history of Raynaud's phenomenon, a well performed barium swallow is probably adequate. Manometry is probably important in patients being considered for surgery with atypical presentations or evidence of Raynaud's disease.

PA: Can you describe any esophageal problems that are not well demonstrated on barium studies or endoscopy but are well suited to a diagnosis by esophageal manometry?

WP: The most important disorder that can be diagnosed manometrically, which may be overlooked by barium studies and endoscopy, is achalasia. We have recently reported that a significant minority of patients with manometrically confirmed achalasia will not have the classic barium $\mathrm{x}$-ray findings. Thus, patients with persisting troublesome dysphagia, without evidence of mechanical obstruction (and this would include negative biopsies for eosinophilic esophagitis), should have esophageal manometry. On the other hand, it is worth pointing out that esophageal manometry does lack sensitivity. There are many patients with normal stationary manometry testing who have evidence of nonspecific contraction abnormalities during videofluoroscopy.

PA: It seems that there is less interest in esophageal manometry among community gastroenterologists. Is this because it has not been considered to be useful? Is it related to remuneration or inadequate training? Are manometry specialists at risk of becoming an extinct species?

WP: I think the enthusiasm that was present 10 years ago surrounding motility disorders and motility testing in general has been waning. This is perhaps largely due to the fact that treatment for esophageal motor disorders has not kept pace with our ability to diagnose them. A diagnosis of achalasia on manometry leads to very specific and useful therapies. Unfortunately, we do not have validated treatments for various other motor disorders. Another factor I think is that trainees and training programs seem to be overwhelmed with procedures and consultations at the expense of other aspects of our specialty, including manometry. Remuneration may also be a factor. It probably takes about the same time to perform a colonoscopy than it does to perform a supervised manometry. The fee for colonoscopy is almost three times greater. However, I do not think that motility specialists are at risk of becoming extinct yet. I suspect motility testing will make a comeback once better treatments are available. I am optimistic that a time will come when we will use drugs to treat functional esophageal disorders that are tailored to the specific motor or sensory abnormality that is detected.

PA: Can you describe some of the new technologies in the assessment of esophageal diseases?

WP: There are a number of new techniques to study esophageal function. High-resolution manometry uses a 36-channel solid state catheter and sophisticated computer software. By vastly increasing the number of recording sites and decreasing the spacing between them, one is able to markedly increase the sensitivity of esophageal manometry. The data are displayed in beautiful isobaric contour plots that give high temporal and spatial resolution. While this will pick up subtle abnormalities that can be missed with routine perfusion manometry, it is yet unclear whether this will improve outcome for our patients. Some laboratories also have the so-called Bilitech 2000 (Medtronic of Canada Ltd) which is capable of detecting when bilirubin makes contact with its sensor. This has been used to assess the degree of bile refluxing into the esophagus. Unfortunately, this device is somewhat inaccurate and has yet to show any clinical benefit.

There has also been a lot of interest in recent years in sensory testing using either a barostat device or an impedance planimetry balloon. These devices are very useful to study pathophysiology and can ascertain whether the patient's symptoms are related to a sensory disorder. This in turn may guide therapy toward drugs that interfere with visceral sensation, such as tricyclic antidepressants. However, because sensory problems are so prevalent, empirical treatment is certainly a reasonable option to this sort of testing.

Finally, esophageal impedance monitoring is now being used in many centres as an adjunct to standard manometry and ambulatory $\mathrm{pH}$ testing. The impedance catheter detects changes in electrical resistance between a series of paired electrodes, thereby detecting movement of air, solids and liquids in either direction within the esophagus. It therefore provides a useful measure of bolus transport during peristalsis, as well as both air and liquid reflux coming from the stomach. When combined with $\mathrm{pH}$ monitoring, it is able to detect 'nonacid' reflux episodes. There is now a growing body of literature suggesting that a small subgroup of patients, with persisting symptoms following PPI therapy, have symptoms caused by nonacid reflux events.

\section{REFERENCES}

1. Paterson WG. Canadian Association of Gastroenterology practice guidelines: Management of noncardiac chest pain.

Can J Gastroenterol 1998;12:401-7.

2. Kahrilas PJ, Clouse RE, Hogan WJ. American Gastroenterological Association technical review on the clinical use of esophageal manometry. Gastroenterology 1994;107:1865-84.

3. Balaban DH, Yamamoto Y, Liu J, et al. Sustained esophageal contraction: A marker of esophageal chest pain identified by intraluminal ultrasonography. Gastroenterology 1999;116:29-37.

4. Pandolfino JE, Kahrilas PJ; American Gastroenterological Association. AGA technical review on the clinical use of esophageal manometry. Gastroenterology 2005;128:209-24.

5. El-Takli I, O'Brien P, Paterson WG. Clinical diagnosis of achalasia: How reliable is the barium x-ray? Can J Gastroenterol 2006;20:335-7. 


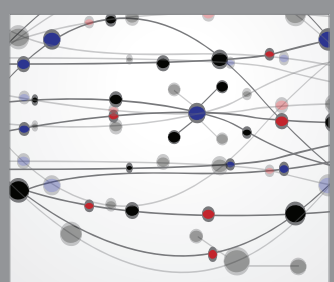

The Scientific World Journal
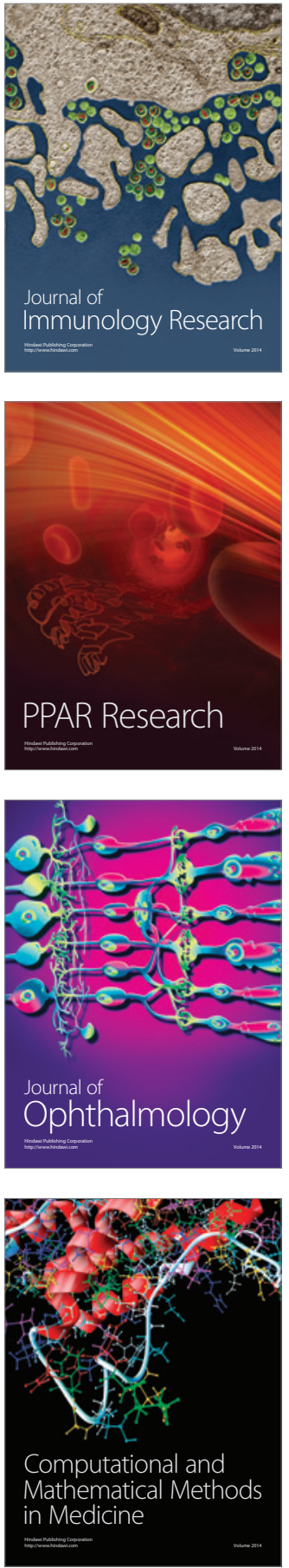

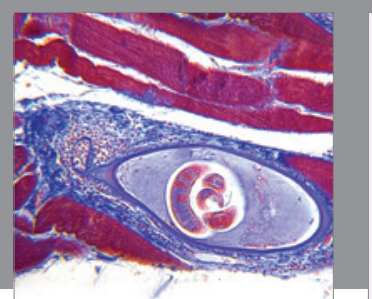

Gastroenterology Research and Practice

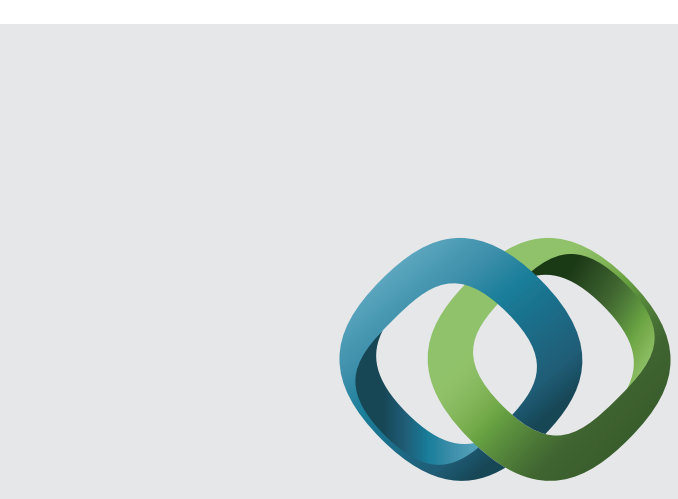

\section{Hindawi}

Submit your manuscripts at

http://www.hindawi.com
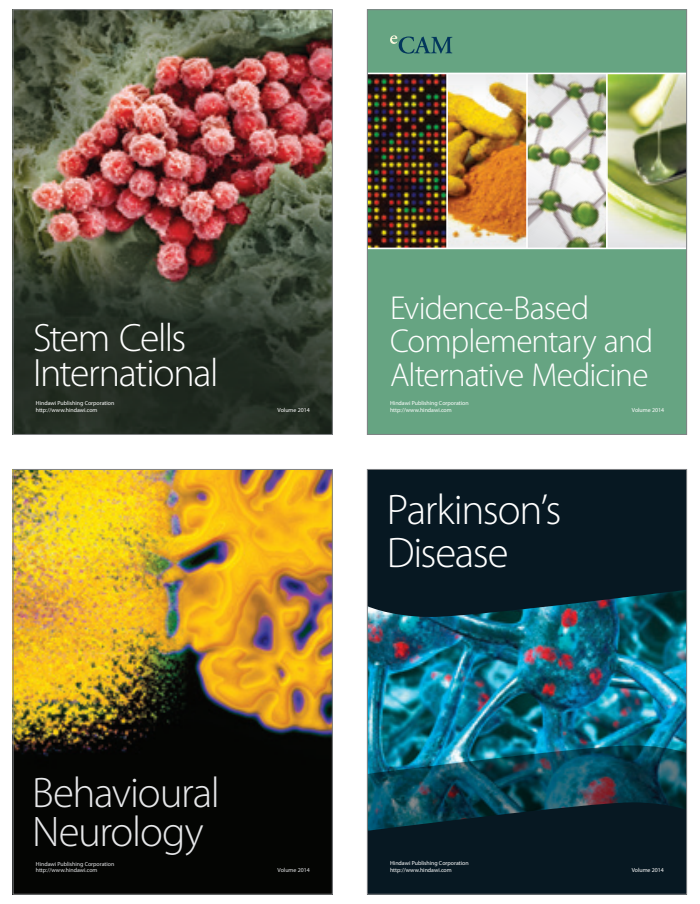
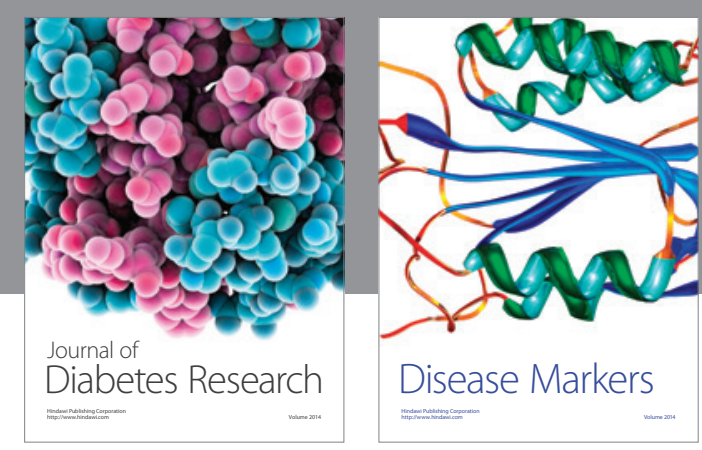

Disease Markers
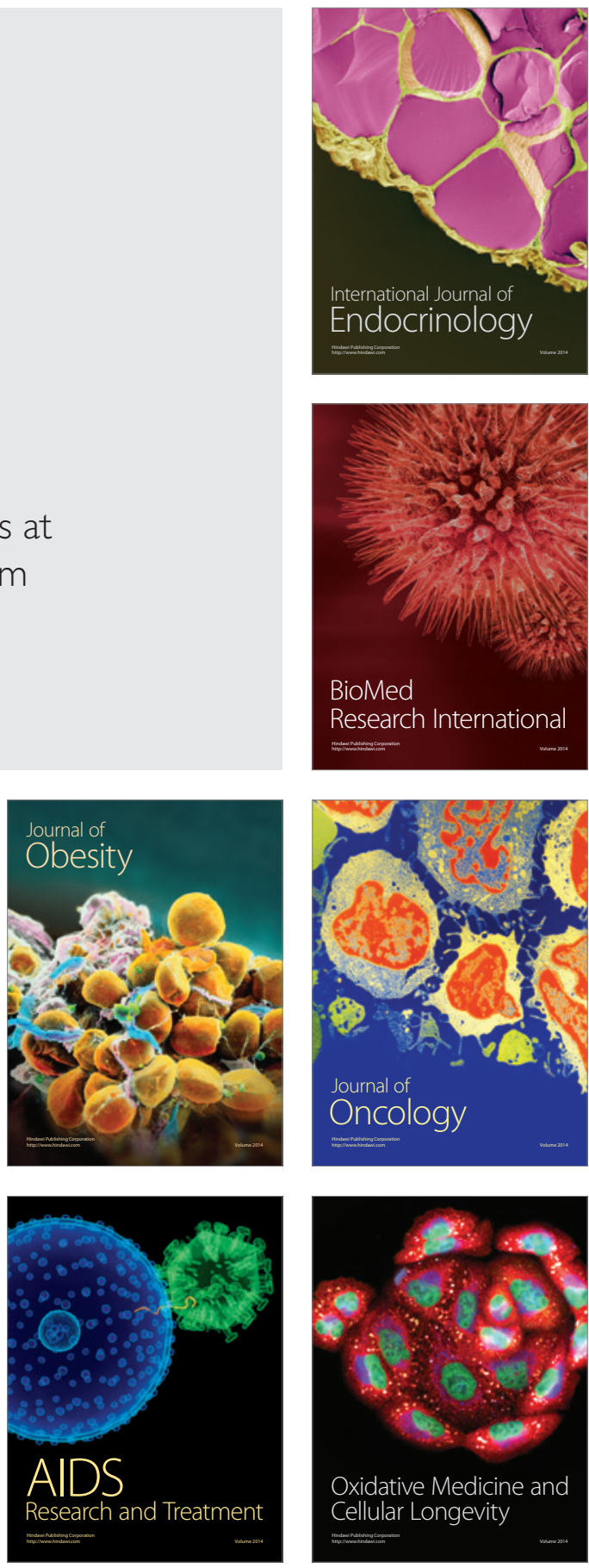